Service social

\title{
Quand l'homophobie se superpose à la discrimination ethnoculturelle
}

\section{Vincent Larouche}

Volume 56, numéro 1, 2010

URI : https://id.erudit.org/iderudit/039777ar

DOI : https://doi.org/10.7202/039777ar

Aller au sommaire du numéro

Éditeur(s)

École de service social de l'Université Laval

ISSN

1708-1734 (numérique)

Découvrir la revue

Citer cet article

Larouche, V. (2010). Quand l'homophobie se superpose à la discrimination ethnoculturelle. Service social, 56(1), 31-42. https://doi.org/10.7202/039777ar d'utilisation que vous pouvez consulter en ligne.

https://apropos.erudit.org/fr/usagers/politique-dutilisation/ 


\title{
Quand 1'homophobie se superpose à la discrimination ethnoculturelle
}

\author{
Vincent LAROUCHE \\ Étudiant en médecine, Université Laval
}

\section{INTRODUCTION}

Au cours des dernières décennies, l'immigration internationale a été en constante évolution et en progression au Québec, les immigrants constituant à ce jour une population de près de $700000^{1}$ personnes, soit environ $9,9 \%{ }^{2}$ de la population québécoise totale. Dans ce contexte, le Québec a comme idéal de devenir une véritable mosaïque ethnoculturelle tolérante et ouverte d'esprit où tous continuent de porter leur culture d'origine, tout en développant leur identité québécoise. Cependant, dans la foulée de la commission Bouchard-Taylor de 2007-2008, une consultation publique sur les accommodements raisonnables, il appert qu'il reste un travail important à faire pour que les immigrants et plus généralement les communautés ethnoculturelles soient véritablement intégrés. Parallèlement à cela, la législation ainsi que les mentalités et valeurs québécoises et canadiennes ont rapidement progressé depuis les années 1970 par rapport au statut de l'homosexualité et des communautés gaies, lesbiennes et bisexuelles, passant de la notion que l'acte homosexuel était un crime jusqu'à une reconnaissance de droits égaux pour les gais et lesbiennes dans les années 2000, le mariage civil et l'adoption leur étant maintenant ouverts. En superposant ces deux sujets, une question épineuse tracasse donc de plus en plus les ressources communautaires des gais et lesbiennes: qu'en est-il de la situation des personnes de la diversité sexuelle issues de milieux ethnoculturels? Comme on sait qu'il existe une différence notable entre la société québécoise et les cultures des immigrants quant à leur attitude face à l'homosexualité, que vit ce groupe, a-t-il accès à des ressources adaptées, quelles difficultés vit-il? Cet essai se veut un portrait global de la situation des lesbiennes, gais, bisexuels et personnes transgenres (LGBT) issus de minorités visibles ou de communautés culturelles au Québec. Les lectures principales qui m'ont permis de dresser un portrait de la situation incluent le rapport de 2007 du Groupe de travail mixte contre l'homophobie, le mémoire de Wong et al. de 2007 sur la diversité sexuelle chez les minorités visibles, les communautés culturelles et les personnes bispirituelles au Québec ainsi que le mémoire conjoint de la

1. Fondation Émergence et de Gai Écoute (2007). Homosexualité et différences culturelles, une crainte raisonnable, Mémoire présenté à la Commission de consultation sur les pratiques d'accommodement reliées aux différences culturelles, Montréal, septembre.

2. Direction de la population et de la recherche du Ministère des relations avec les citoyens et de l'immigration (2003). Caractéristiques de l'immigration au Québec. [http://www.micc.gouv.qc.ca/publications/fr/planification/0507-caracteristiques-immigration.pdf] (page consultée le 8 décembre 2009). 
Fondation Émergence et de Gai Écoute de 2007 sur l'homosexualité en lien avec les différences culturelles. Mes recherches ont également été complétées par la lecture d'articles tirés de journaux, de revues d'intérêt gai, et la consultation de sites Internet, entre autres du ministère québécois des Relations avec les citoyens et de I'Immigration, de la Fondation Émergence, du Patrimoine canadien. Je traiterai d'abord dans ce texte des problèmes personnels vécus par les LGBT, ce point incluant des exemples concrets de perspectives culturelles sur l'homosexualité, l'impact de ces perspectives sur leur vie ainsi que l'évolution des mentalités des immigrants de deuxième génération, puis j'aborderai les problèmes collectifs vécus par les personnes de la diversité sexuelle issues des minorités culturelles, tels que la double stigmatisation qu'elles vivent, le manque de visibilité médiatique dont elles sont victimes et les procédures d'immigration. Je terminerai en recensant les ressources qui leur sont accessibles et les initiatives à leur intention et en proposant des pistes de solution aux difficultés rencontrées par ce groupe à risque. Cet essai s'adresse aux travailleurs sociaux et autres professionnels de la santé ainsi qu'à tous ceux et celles désirant être plus au fait des difficultés propres aux personnes de la diversité sexuelle issues de minorités ethnoculturelles.

\section{PROBLÈMES PERSONNELS VÉCUS PAR LES PERSONNES DE LA DIVERSITÉ SEXUELLE ISSUES DE MINORITÉS ETHNOCULTURELLES}

\section{Exemples concrets de perspectives culturelles sur l'homosexualité}

Alors que le Québec comme province et le Canada comme pays ont été des pionniers en matière d'avancement des droits des gais et lesbiennes, l'homosexualité est encore illégale et punissable de sévères châtiments (voire de mort par pendaison dans plusieurs États du Moyen-Orient) dans plus de 70 pays. Toutefois, la Charte canadienne des droits et libertés, plus spécifiquement son article 15, stipule que :

La loi ne fait acception de personne et s'applique également à tous, et tous ont droit à la même protection et au même bénéfice de la loi, indépendamment de toute discrimination, notamment des discriminations fondées sur la race, l'origine nationale ou ethnique, la couleur, la religion, le sexe, l'âge, les déficiences mentales ou physiques et l'orientation sexuelle ${ }^{3}$.

De ce fait, l'orientation sexuelle est un motif interdit de discrimination au Canada. Afin d'illustrer concrètement la discordance entre cette valeur nationale et la vision d'autres cultures, voici quelques exemples de la perception de l'homosexualité dans les cultures des pays d'origine d'immigrants québécois. Dans certaines communautés de Montréal, l'homosexualité est taboue et est perçue comme une menace à l'identité de la collectivité ${ }^{4}$. Ainsi, un jeune Montréalais, président de Jeunesse noire en action,

3. Patrimoine canadien. Article 15 - Droits à l'égalité.

[http://www.pch.gc.ca/pgm/pdp-hrp/canada/guide/eql-egl-fra.cfm] (page consultée le 8 décembre 2009).

4. McGill University Student Health Service, Gays, Lesbians, Bisexuals and Race. [http://www.mcgill.ca/studenthealth/information/queerhealth/glbrace/] (page consultée le 8 décembre 2009). 
expliquait en entrevue : «Pour une personne gay et noire, développer une identité est très difficile. Pour bien des gens, être noir et être gay, ce n'est pas compatible. Dans la communauté noire, être gay, c'est être blanc ${ }^{5}$. " Semblablement, certains groupes latino-américains mettent en relief la primauté de l'hétérosexualité et leur culture est fortement fondée sur une division rigide des rôles socio-sexuels. Une conséquence directe de cette vision est la désapprobation de tout comportement social ou sexuel qui ne correspond pas à la division traditionnelle des rôles socio-sexuels. Dans la communauté libanaise, des personnes soupçonnées d'homosexualité sont considérées avoir "enfreint l'honneur de la famille » et courent le risque d'être assassinées lors d'un séjour au Liban, racontent de jeunes immigrants libanais. Au chapitre religieux, un point sur lequel s'entendent les trois grandes religions monothéistes, soit le christianisme, le judaïsme et l'islamisme, est que l'homosexualité serait un péché « contre nature ». Des jeunes gais sont condamnés à mort dans des pays islamiques intégristes tels que l'Arabie Saoudite et l'Iran. Le Coran en tant que tel reste vague sur l'homosexualité, il n'en mentionne pas le terme, mais une certaine interprétation de l'épisode de Loth et du peuple de Sodome (peuple ayant, selon la quatrième sourate, inventé I'homosexualité et ayant également été puni par Allah par un déluge de feu et de soufre) fait prévaloir une vision fortement condamnatrice ${ }^{6}$. Bien que les musulmans immigrés soient souvent plus modérés sur le plan religieux, la réalité des jeunes gais musulmans au Québec reste ardue. Dans une entrevue faite par un journaliste d'un magazine d'intérêt homosexuel, un jeune de descendance marocaine explique: «Faire son coming-out, c'est toujours difficile, mais ça l'est encore plus quand on est musulman. Dans la société maghrébine, l'homosexualité est taboue. Quand on en parle, on est tout simplement exclu. Mes parents sont musulmans, mais ne pratiquent pas beaucoup, alors ce fut plus facile de leur dire. $\mathrm{Ce}$ n'est toutefois pas un sujet que nous abordons souvent ${ }^{7}$. " Alors que les cultures autochtones intégraient l'homosexualité et la bisexualité et que les «personnes-auxdeux-esprits ${ }^{8} \gg$ avaient des rôles importants, étaient respectées et positivement perçues comme une richesse par leurs traditions ancestrales, il règne actuellement dans plusieurs Premières Nations une homophobie qui découle de la colonisation européenne et de l'évangélisation forcée de communautés autochtones que les jeunes gais et lesbiennes ont tendance à quitter.

\section{L'impact de ces perceptions sur la vie des personnes de la diversité sexuelle issues de minorités ethnoculturelles}

Tel qu'effleuré lors du témoignage du jeune Marocain, la réalité des personnes de la diversité sexuelle issues de communautés ethnoculturelles fait en sorte que le dévoilement de leur orientation sexuelle s'effectue dans un contexte de peur, d'hostilité

5. Elkouri Rima (2001). «Quand racisme et homophobie se conjuguent », La Presse, 29 août 2001, p. B1.

6. Lafontaine, Yves (2002). Que dit le Coran de l'homosexualité? [http://www.fugues.com/main.cfm?l=fr\&p=100_Article\&article_id=678\&rubrique_ID=81]. (page consultée le 8 décembre 2009).

7. Lafontaine, Yves (2002). Vivre sa vie... différemment. [http://www.fugues.com/main.cfm?l=fr\&p=100_Article\&article_id=672\&rubrique_ID=81] (page consultée le 8 décembre 2009).

8. Nom donné aux personnes autochtones non-conformistes sur le plan du sexe et du genre. Ayant à la fois du masculin et du féminin, elles sont présumées être inspirées par deux esprits. 
et de violence réelle ou anticipée. Ce «coming-out » peut même être reçu comme une trahison, un reniement des valeurs culturelles traditionnelles. II est fréquent que les gais, lesbiennes et bisexuels des communautés culturelles soient rejetés par leur famille proche et leur communauté. Une fois leur orientation sexuelle dévoilée, ces personnes se disent couramment victimes de violence psychologique et physique. Ce climat doublé d'une forte pression sociale à la conformité incite donc les personnes homosexuelles et bisexuelles de minorités culturelles à vivre une double vie et à dissimuler leur orientation sexuelle. De ce fait, certains fréquentent moins les lieux de socialisation des communautés gaies et lesbiennes, d'autres se résignent à vivre leur homosexualité en dehors des milieux lesbiens et gais, dans une stratégie d'invisibilité. De plus, cette même population hésite à faire appel aux organismes communautaires, au réseau de la santé et des services sociaux ou aux autres services publics qui leur sont offerts, appréhendant le dévoilement de leur orientation sexuelle et ses conséquences possibles sur leur réalité quotidienne. En conséquence, ces gens se sentent souvent isolés et peu intégrés socialement, vivent une tension psychologique continue et sont plus à risque relativement au suicide et à la consommation de drogues illicites. Les « personnes-aux-deux-esprits » vivent une situation similaire; elles doivent souvent s'expatrier de leur communauté autochtone et sont plus à risque de développer des polytoxicomanies et autres problèmes de «fuite en avant ». Dans un article sur les hommes issus de communautés hispaniques d'Amérique, Pierre $\mathrm{J}$. Tremblay rapporte :

Nous avons observé que les idées suicidaires sont plus fréquentes chez les homosexuels issus de minorités raciales ou ethniques que chez les homosexuels blancs. [...] En général, une minorité dépréciée au sein d'une autre peut être plus encline aux tendances suicidaires. Par exemple, les membres homosexuels d'une minorité ethnique sont souvent reniés par leurs propres institutions sociales et celles de la majorité $[\ldots]^{9}$.

La situation des personnes de la diversité sexuelle appartenant à des groupes culturels minoritaires est souvent peu reluisante et nombre de défis supplémentaires se dressent sur le chemin du dévoilement de leur orientation sexuelle. Une réaction fréquente de ces jeunes est donc de prendre leurs distances vis-à-vis de leur communauté d'origine et de chercher le soutien de communautés gaies et lesbiennes estimées (paradoxalement) plus majoritaires.

9. Tremblay, Pierre J. « The Additional Problems of Gay, Lesbian, and Bisexual Youth of Colour », dans The Gay, Lesbian, and Bisexual Factor in the Youth Suicide Problem.

[http://www.sws.soton.ac.uk/gay-youth-suicide/04-gay-youth-of-colour.htm] (page consultée le 8 décembre 2009). 


\section{Espoir à l'horizon : l'évolution des mentalités des immigrants de deuxième génération}

Dans cette courte section, je résumerai les grandes lignes d'un sondage québécois présenté lors de la Journée internationale contre l'homophobie de $2009^{10}$, témoignant d'une évolution positive de la perception de l'homosexualité chez les immigrants québécois de seconde génération. Alors que $48 \%$ des immigrants de première génération croient que l'homosexualité est une maladie ou un comportement sexuel déviant, c'est seulement le cas de $24 \%$ de leurs enfants. Tandis que $62 \%$ des parents qualifieraient l'homosexualité d'aussi valable que d'autres orientations sexuelles, c'est le cas de $86 \%$ des immigrants de deuxième génération. Pour la moitié des immigrants de première génération, l'homosexualité est «normale », alors que $71 \%$ de leurs descendants la qualifient ainsi. Cinquante-six pour cent (56\%) des immigrants de première génération ont avoué qu'ils seraient honteux de dévoiler à leur famille qu'un de leurs enfants est homosexuel(le), tandis que $81 \%$ de leurs enfants ont déclaré qu'ils n'auraient pas ce sentiment. Dans le même ordre d'idées, les immigrants de seconde génération se montraient beaucoup plus ouverts que leurs parents dans le domaine du droit au mariage des couples de même sexe et plus nombreux à considérer que la discrimination basée sur l'orientation sexuelle est inacceptable. De plus, la proportion d'enfants d'immigrants ayant déclaré connaître personnellement des gais et lesbiennes était substantiellement plus grande que cette même proportion chez leurs parents. Ce sondage représente bien l'évolution des mentalités et l'ouverture d'esprit des enfants d'immigrants. Aux yeux du président de la Fondation Émergence : « Cela signifie qu'à l'égard de l'homosexualité, les immigrants de deuxième génération soutiennent les valeurs québécoises d'égalité plus que leurs parents. Dans le cas où plusieurs de ces parents viennent de pays qui punissent des gens en raison de leur orientation sexuelle, c'est un vrai plaisir de découvrir que ces jeunes abandonnent certaines valeurs alors qu'ils adoptent celles de leur société adoptive ${ }^{11}$. »

\section{PROBLÈMES COLLECTIFS VÉCUS PAR LES PERSONNES DE LA DIVERSITÉ SEXUELLE ISSUES DE MINORITÉS ETHNOCULTURELLES}

\section{Double stigmatisation et discrimination au sein de la communauté homosexuelle}

Le sentiment d'appartenance à une communauté est un besoin intrinsèque de l'existence humaine. Paradoxalement, alors que l'on serait porté à penser qu'en raison de l'oppression et de la discrimination homophobes dont sont victimes les gais, lesbiennes et bisexuels, ils sont généralement eux-mêmes plus tolérants et ouverts d'esprit et ne reproduisent pas les mêmes mécanismes d'exclusion que ceux contre lesquels ils se battent, il appert plutôt que les homosexuels issus de minorités ethnoculturelles font également face à la xénophobie et au racisme dans les milieux gais et lesbiens. En fait, l'incidence des comportements discriminatoires à l'endroit des

\footnotetext{
10. Fondation Émergence (2009). Significant Generation Gap Between Immigrants - Perception of Homosexuality. [http://gaiecoute.org/default.aspx?scheme=3751] (page consultée le 8 décembre 2009).

11. Ibid.
} 
personnes des communautés culturelles chez les gais et lesbiennes serait comparable à celle observée dans la société québécoise en général ${ }^{12}$. Un des facteurs de cette discrimination est l'ignorance des autres cultures, qui génère des jugements défavorables sur les membres de ces minorités. Des exemples très crus de tels préjugés ethniques incluent par exemple l'idée que les jeunes hommes asiatiques ou arabes sont des partenaires sexuels serviles et soumis, réduisant abjectement les jeunes homosexuels de communautés culturelles à des objets sexuels exotiques. D'où le concept de double stigmatisation: les personnes de la diversité sexuelle issues de minorités visibles sont à la fois victimes d'homophobie au sein de leur communauté d'origine et de discrimination ethnoculturelle au sein de la communauté homosexuelle (les lesbiennes peuvent même être victimes d'une triple discrimination, considérant la misogynie qui est encore omniprésente dans certaines cultures). Tous ces facteurs nuisent fortement à l'intégration de cette population et à leur impression d'être des citoyens québécois à part entière. Un exemple de cette incompréhension ethnocentriste réside en l'encouragement systématique à la révélation de son orientation sexuelle, critère d'adhésion à un organisme communautaire LGBT excluant alors une bonne partie des membres des minorités culturelles; le modèle de vie homosexuelle « ouverte » est associé au contexte occidental, voire nord-américain. Les organismes communautaires gais et lesbiens reconnaissent franchement être peu sensibilisés aux problèmes d'exclusion des personnes de minorités sexuelles des communautés culturelles, quoique quelques organismes aient commencé à se pencher sur la question dans l'objectif de les sortir de leur isolement et de sensibiliser les communautés culturelles aux droits des personnes de la diversité sexuelle.

\section{Manque de visibilité médiatique et de modèles}

En corollaire, un autre obstacle à l'intégration réelle des personnes de la diversité sexuelle issues de minorités culturelles est leur déplorable absence de représentation au sein des principaux organismes communautaires et des médias homosexuels. En effet, il est logique de croire qu'une visibilité accrue au sein de magazines, d'émissions de radio et de télévision ou de sites Internet d'intérêt homosexuel pourrait contribuer à enrayer les préjugés et idées préconçues et à intégrer davantage les gais, lesbiennes, bisexuels de minorités ethnoculturelles à la communauté homosexuelle ou lesbienne. Dans cette même lancée, de puissants vecteurs culturels tels les films, romans, téléséries ou livres actuels qui abordent l'homosexualité et la bisexualité dépeignent en général ces réalités comme des phénomènes liés à une société blanche européenne et nord-américaine; lorsque des personnages homosexuels de milieux ethnoculturels sont présents, il s'agit trop souvent de caricatures promouvant la généralisation de stéréotypes. Concomitamment au manque de visibilité médiatique, un autre problème s'impose aux personnes de la diversité sexuelle issues de minorités culturelles: le manque flagrant de modèles gais et lesbiens issus de leur communauté. Pour des jeunes et des adultes gais et lesbiennes, cette absence de modèles autour d'eux rend difficile le dévoilement ou l'acceptation personnelle de leur orientation sexuelle. Le jeune président de Jeunesse noire en action cité plus tôt posait la question suivante : « Comment s'assumer pleinement quand personne autour de soi n'ose le faire? » Alors

12. Groupe de travail mixte contre l'homophobie (2007). De l'égalité juridique à l'égalité sociale: Vers une stratégie nationale de lutte contre l'homophobie, Rapport, Québec, Commission des droits de la personne et des droits de la jeunesse. 
que les médias et l'entourage quotidien des gais, lesbiennes et bisexuels issus de minorités ethnoculturelles semblent rejeter leurs attributs culturels ou même carrément nier leur existence, des situations épineuses peuvent survenir, comme dans le cas de femmes musulmanes pratiquantes, lesbiennes ou bisexuelles qui, portant couramment le voile pour des raisons religieuses, politiques et personnelles, sont rejetées par les communautés gaies et lesbiennes puisque considérées comme des freins à l'avancement des droits homosexuels.

\section{Le choc de l'immigration}

Le sort réservé aux personnes de la diversité sexuelle est encore dangereux dans plus de 70 pays. L'homosexualité est un crime punissable de la peine de mort ou de la prison à perpétuité dans une dizaine d'entre eux et est illégale dans plusieurs autres. Le Québec et le Canada accueillent des immigrants en provenance de tels pays. Immanquablement, ces divergences dans les valeurs peuvent créer des distorsions notables en ce qui a trait aux demandes d'immigration. Un exemple concret est, dans le processus de sélection des candidats à l'immigration, le traitement uniforme accordé par le ministère de l'Immigration et des Communautés culturelles aux demandes des conjoints de fait de même sexe et de sexes différents. Chez les conjoints de fait hétérosexuels aussi bien qu'homosexuels, le Ministère examine si les deux candidats satisfont à la définition de conjoints de fait, selon le Règlement sur la sélection des ressortissants étrangers ${ }^{13}$ qui stipule que des preuves de cohabitation, partage des biens, connaissance de la relation par le reste de la famille et des documents légaux doivent être fournis. Or, ces critères constituent un obstacle considérable, puisque de telles preuves exposeraient bon nombre de requérants à des sanctions pénales et aux hostilités homophobes qui existent dans leur pays d'origine. En outre, plusieurs immigrants estiment que les services d'aide à l'intégration sont hétérosexistes et que les agents et intervenants de ces services sont plutôt démunis de connaissances face à la réalité homosexuelle. De l'autre côté de la médaille, les personnes qui immigrent au Québec reçoivent peu d'information sur les principaux motifs de discrimination interdits au Québec (réitérons ici que l'orientation sexuelle fait partie de ces derniers). De plus, malgré le fait que le programme de formation linguistique du ministère de l'Immigration et des Communautés culturelles contient une explication aux immigrants des valeurs de la société québécoise, notamment l'égalité des personnes et les droits garantis par la Charte des droits et libertés de la personne du Québec ${ }^{14}$, la thématique de l'égalité des droits (mariage, union civile, adoption, etc.) des personnes homosexuelles serait souvent omise ou vaguement mentionnée. Bref, tant au niveau de la sélection des immigrants que de l'information qui leur est fournie sur la société québécoise, les méthodes du ministère de l'Immigration et des Communautés culturelles sont peu adaptées à la réalité homosexuelle.

13. Gouvernement du Québec (2005). Règlement sur la sélection des ressortissants étrangers, L.R.Q., c. I-0.2, r.5, Québec, Éditeur officiel du Québec.

14. Gouvernement du Québec. Charte des droits et libertés de la personne, L.R.Q., c. C-12. 


\section{RESSOURCES, INITIATIVES ET SOLUTIONS}

\section{Ressources actuelles visant les personnes de la diversité sexuelle issues de minorités ethnoculturelles}

Depuis quelques années, plusieurs organismes communautaires spécialisés à l'intention des personnes de la diversité sexuelle de groupes culturels spécifiques ont vu le jour : AIDS Community Care Montreal (vise à améliorer la qualité de vie des gens séropositifs, surtout de la communauté anglophone de Montréal), Afrique Arc-en-Ciel (cible la diaspora québécoise des gais, lesbiennes, bisexuels et transsexuels d'Afrique et des Caraïbes), l'Association canadienne des personnes bispirituelles (s'adresse aux gais, lesbiennes, bisexuels et transgenres des Premières Nations canadiennes), Feygelah et Ga'ava (groupes de soutien anglophones pour la communauté juive homosexuelle), GLAM (groupe social activiste offrant du soutien aux homosexuels asiatiques de Montréal), Helem (se définit comme un organisme de protection des homosexuels d'origine libanaise face à la persécution, la discrimination et l'ignorance de la réalité homosexuelle au sein de cette communauté), HOLA (groupe de soutien destiné aux hommes gais hispanophones), le Gruppo Italiano Gay e Lesbico di Montreal (offre du soutien aux homosexuels italo-québécois), Zaafaran (espace d'entraide pour femmes lesbiennes, bisexuelles et transgenres d'origine maghrébine ou du Moyen-Orient), et d'autres encore. De plus, des organismes communautaires généraux pour gais, lesbiennes et bisexuels, tels Gai Écoute, Fondation Émergence, Jeunesse Lambda, Projet 10, Séro-Zéro, GRIS ou encore AlterHéros, peuvent offrir de l'écoute, du soutien, des ressources aux allosexuels ${ }^{15}$ par rapport à leurs questionnements et leurs expériences de vie en ce qui a trait à leur orientation sexuelle, mais leur approche n'est pas forcément adaptée aux personnes issues de milieux ethnoculturels. Malheureusement, ainsi que le souligne le Rapport de consultation du Groupe de travail mixte contre l'homophobie en 2007, bon nombre de groupes des communautés gaies et lesbiennes œuvrant auprès des personnes de minorités culturelles ne bénéficient d'aucun soutien financier (gouvernemental, privé ou autre). Autre point négatif, ces groupes sont pour la plupart basés à Montréal, les gais et lesbiennes de minorités culturelles ailleurs en province disposant de peu de ressources, et la majorité visent les adolescents ou jeunes adultes et plus souvent les hommes que les femmes, sélectivité qui laisse de nombreuses personnes (femmes, adultes plus vieux, etc.) sans soutien.

\section{Initiatives positives afin de soutenir les personnes de la diversité sexuelle issues des minorités ethnoculturelles}

Comme une proportion notable d'organismes communautaires, y compris LGBT, les organismes de soutien visant les gais, lesbiennes et bisexuels des communautés ethnoculturelles n'ont qu'un maigre soutien financier des gouvernements provinciaux et fédéraux, les limitant souvent fortement dans leur mission d'aide et d'amélioration de la condition de ces groupes. Néanmoins, des initiatives positives voient le jour afin de combler ces lacunes. En effet, à la suite du dévoilement par la Fondation Émergence du sondage québécois mentionné plus haut qui fait état de l'étendue des préjugés

15. Terme désignant toutes les personnes non exclusivement hétérosexuelles. 
homophobes dans les communautés d'immigrants (préjugés étant plus présents chez les immigrants de première génération), le 15 mai 2009, Yolande James, ministre québécoise de l'Immigration et des Communautés culturelles, a annoncé un investissement de 150000 \$ pour contrer l'homophobie chez les immigrants ${ }^{16}$. Dans un spectre d'action plus large, le 11 décembre 2009, Kathleen Weil, ministre québécoise de la Justice et ministre responsable de la lutte contre l'homophobie, a déposé la Politique québécoise de lutte contre l'homophobie, "l'une des pièces maîtresses d'une stratégie plus large devant mener à la reconnaissance pleine et entière des personnes de minorités sexuelles, à leur soutien sur les plans institutionnel et communautaire et à l'amélioration des connaissances relatives à la diversité sexuelle $^{17} »$. Entre autres, cette politique mentionne la nécessité d'adapter les services sociaux, de santé et communautaires actuels aux problèmes vécus par les personnes de minorités culturelles et sexuelles, en restant cependant vague sur les solutions possibles.

\section{Pistes de solution pour améliorer la réalité des personnes de la diversité sexuelle issues de minorités ethnoculturelles}

Les pistes de solution proposées ci-après superposent des initiatives originales et des recommandations tirées du Rapport de consultation du Groupe de travail mixte contre l'homophobie (2007) ainsi que du mémoire de Wong et al. (2007). D'abord, je crois qu'afin de lutter contre certaines perspectives culturelles fortement négatives à l'égard de l'homosexualité, des organismes communautaires gais, lesbiens et bisexuels devraient monter des campagnes de sensibilisation (peut-être sous forme d'ateliers interactifs) qui seraient destinées à des communautés culturelles précisément ciblées. Par exemple, des ateliers pourraient être tenus lors des réunions de groupes de défense des minorités noires, latino-américaines, arabes, etc. Dans la même lancée, et avec l'aide d'historiens de ces nations, des ateliers interactifs pourraient être dispensés dans des écoles secondaires ou des centres communautaires de communautés autochtones afin d'y redorer le blason des «personnes-aux-deux-esprits » et de favoriser leur réintégration dans les mœurs de ces collectivités. Par ailleurs, il serait primordial que le Secrétariat aux affaires autochtones se dote de mesures de protection des personnes bispirituelles contre l'homophobie. En ce qui a trait à la discrimination ethnoculturelle omniprésente dans les communautés gaies, lesbiennes et bisexuelles, une visibilité accrue des personnes de la diversité sexuelle issues de minorités ethnoculturelles dans les médias d'intérêt homosexuel pourrait atténuer certains préjugés et idées préconçues racistes et xénophobes. Cela pourrait se faire par exemple via des chroniques culturelles hebdomadaires esquissant la situation des homosexuels dans différentes régions du monde ou présentant les expériences d'immigrants venus au Québec par rapport à la diversité sexuelle. Créer des personnages authentiques gais, lesbiens ou bisexuels issus de minorités visibles dans des films, livres, et téléséries viserait ce même objectif. Des campagnes de

16. Point de Bascule (2009). Homophobie chez les immigrants : Québec investira $150000 \$$. [http://www.pointdebasculecanada.ca/breve/2066-quebec-homophobie-chez-les-immigrants-quebecinvestira-150-000.php] (page consultée le 8 décembre 2009).

17. Ministère de la justice du Québec (2009). Politique québécoise de lutte contre l'homophobie. [http://www.justice.gouv.qc.ca/FRANCAIS/publications/rapports/pdf/homophobie.pdf] (page consultée le 7 avril 2010). 
sensibilisation favorisant les échanges interculturels devraient également être conçues et intégrées dans des médias d'intérêt gai, lesbien, bisexuel, prenant par exemple la forme d'annonces accrocheuses du type «Les LGBT ne sont pas que roses... » ou encore « II n'y a pas que l'arc-en-ciel qui ait plusieurs couleurs... ». De plus, des organismes communautaires pourraient concevoir des espaces de rencontre multiculturelle pour les personnes de la diversité sexuelle, par exemple une maison des jeunes ou un «drop-in centre » pour gais, lesbiennes, bisexuels de toutes provenances où des travailleurs sociaux seraient accessibles et où, à intervalles réguliers, des rencontres d'information et des groupes de soutien spécifiques seraient offerts aux gais, lesbiennes et bisexuels des différentes origines ethnoculturelles (ex. mardi soir, asiatiques et leurs sympathisants). Quant aux organismes communautaires pour gais, lesbiennes et bisexuels, ils devraient revoir leurs critères afin d'éviter l'ethnocentrisme, d'assurer une bonne représentativité de leurs intervenants et de permettre à des gens de la diversité sexuelle de minorités culturelles d'avoir des postes clés. Ces organismes devraient également élaborer de nouveaux programmes ou modifier leurs programmes actuels afin d'adapter davantage leurs interventions aux minorités culturelles (par exemple, ajouter un volet spécifique sur la réalité des gais et lesbiennes d'autres groupes culturels dans la formation des écoutants de Gai Écoute). Pour sa part, le gouvernement du Québec, particulièrement le ministère de la Santé et des Services sociaux, devrait prévoir un budget afin de soutenir les organismes déjà en place et de favoriser le développement de nouveaux organismes ayant pour mission la promotion des droits et le soutien des personnes de la diversité sexuelle issues des minorités ethnoculturelles. II serait également pertinent pour ce dernier ministère d'actualiser ses interventions auprès des gais, lesbiennes et bisexuels de communautés culturelles, notamment en mettant sur pied des projets de recherche sur le suicide chez les jeunes de minorités sexuelles et culturelles et l'incidence de l'homophobie dans les communautés ethnoculturelles et autochtones. Pour ce qui est du ministère de l'Immigration et des Communautés culturelles, le Règlement sur la sélection des ressortissants étrangers ${ }^{18}$ devrait revoir les critères qui définissent les conjoints de fait afin que ne soient pas exigées des pièces justificatives potentiellement délétères aux couples de même sexe; le ministère pourrait élaborer une méthode alternative pour statuer sur les demandes de conjoints de fait homosexuels. De plus, les intervenants des services d'immigration devraient recevoir des formations particulières sur les réalités homosexuelles. Dans le même ordre d'idées, il serait primordial que le guide Apprendre le Québec ${ }^{19}$, qui est remis aux immigrants, fasse mention non seulement du fait que l'orientation sexuelle est un motif de discrimination interdit par la loi, mais aussi du fait que le respect et l'égalité des personnes de la diversité sexuelle sont des valeurs québécoises. Dans son programme de formation linguistique, le ministère de l'Immigration et des Communautés culturelles se doit d'inclure la mention du fait que l'égalité des droits des personnes homosexuelles est une priorité pour la société québécoise. Enfin, il est crucial de sensibiliser les autorités juridiques et législatives au fait qu'en concordance avec la Charte canadienne des

18. Règlement sur la sélection des ressortissants étrangers, op. cit.

19. Direction des politiques et programmes d'intÉgration, de rÉgionalisation et de relations interculturelles du MinistÈre de l'immigration et des communautÉs culturelles (2008). Apprendre le Québec : guide pour réussir mon intégration, $2^{\mathrm{e}}$ éd., Québec, Immigration et Communautés culturelles, $166 \mathrm{p}$. 
droits et libertés ${ }^{20}$, des motifs religieux ne devraient jamais être invoqués pour justifier la perpétration d'actes de discrimination homophobes.

\section{CONCLUSION}

Il a été mis en relief qu'au Québec, les personnes de la diversité sexuelle issues de minorités culturelles ou de communautés autochtones sont souvent confrontées aux conceptions homophobes de leur culture d'origine, ce qui entraîne souvent le développement d'une double vie chez plusieurs et augmente leur taux de suicide. II appert également que cette population est généralement victime d'une double stigmatisation, puisque les préjugés racistes et la discrimination ethnoculturelle sont omniprésents dans la communauté homosexuelle. Collectivement, les gais, lesbiennes et bisexuels de minorités ethnoculturelles ont peu de visibilité médiatique et de présence dans les manifestations culturelles québécoises. Par ailleurs, les procédures de sélection et d'information des immigrants et le nombre d'organismes et de ressources de soutien ne sont pas adaptés à leurs besoins; certaines ressources communautaires et instances gouvernementales font néanmoins de petits pas afin de tenter d'améliorer leur sort. Plusieurs pistes de solution ont été proposées pour améliorer la situation des gais, lesbiennes, bisexuels d'origines ethnoculturelles minoritaires au Québec, notamment des campagnes de sensibilisation auprès de la communauté homosexuelle, de divers ministères et de collectivités cibles. Finalement, il faut espérer que concomitamment avec une meilleure intégration des immigrants, la société québécoise devienne réellement une mosaïque multiculturelle où tous tirent le meilleur de la culture de l'autre, à la manière de bon nombre d'immigrants de seconde génération qui semblent délaisser les conceptions homophobes de leur culture d'origine.

\section{BIBLIOGRAPHIE}

DiRECTION DE LA POPULATION ET DE LA RECHERCHE DU MINISTÈRE DES RELATIONS AVEC LES CITOYENS ET DE L'IMMIGRATION. Caractéristiques de l'immigration au Québec. [http://www.micc.gouv.qc.ca/publications/fr/planification/0507-caracteristiquesimmigration.pdf] (page consultée le 8 décembre 2009).

DIRECTION DES POLITIQUES ET PROGRAMMES D'INTÉGRATION, DE RÉGIONALISATION ET DE RELATIONS INTERCULTURELLES DU MINISTĖRE DE L'IMMIGRATION ET DES COMMUNAUTÉS CULTURELLES (2008). Apprendre le Québec : guide pour réussir mon intégration, $2^{\mathrm{e}}$ éd., Québec, Immigration et Communautés culturelles, 166 p.

ElkouRI, Rima. (2001). «Quand racisme et homophobie se conjuguent », La Presse, 29 août 2001, p. B1.

FONDATION ÉMERGENCE ET GAI ÉCOUTE (2007). Homosexualité et différences culturelles, une crainte raisonnable, Mémoire présenté à la Commission de consultation sur les pratiques d'accommodement reliées aux différences culturelles, Montréal, septembre.

20. Ministère de la justice du Canada. Charte canadienne des droits et libertés. [http://lois.justice.gc.ca/fr/charte/1.html] (page consultée le 8 décembre 2009). 
FONDATION ÉMERGENCE (2009). Significant Generation Gap Between Immigrants Perception of Homosexuality [http://gaiecoute.org/default.aspx?scheme=3751] (page consultée le 8 décembre 2009).

Gouvernement DU QuÉBEC. Charte des droits et libertés de la personne, L.R.Q., c. C-12.

GROUPE DE TRAVAIL MIXTE CONTRE L'HOMOPHOBIE (2007). De l'égalité juridique à l'égalité sociale : Vers une stratégie nationale de lutte contre l'homophobie, Rapport, Québec, Commission des droits de la personne et des droits de la jeunesse.

LAFONTAINE, Yves (2002). Que dit le Coran de l'homosexualité? [http://www.fugues.com/main.cfm?|=fr\&p=100_Article\&article_id=678\&rubrique_ID= 81] (page consultée le 8 décembre 2009).

LAFONTAINE, Yves (2002). Vivre sa vie... différemment.

[http://www.fugues.com/main.cfm?l=fr\&p=100_Article\&article_id=672\&rubrique_ID= 81] (page consultée le 8 décembre 2009).

McGill University Student Health Service. Gays, Lesbians, Bisexuals and Race [http://www.mcgill.ca/studenthealth/information/queerhealth/glbrace/] (page consultée le 8 décembre 2009).

MINISTÈRE DE LA JUSTICE DU QUÉBEC. Politique québécoise de lutte contre I'homophobie

[http://www.justice.gouv.qc.ca/FRANCAIS/publications/rapports/pdf/homophobie.pdf ] (page consultée le 7 avril 2010).

PATRIMOINE CANADIEN. Article 15 - Droits à l'égalité. [http://www.pch.gc.ca/pgm/pdphrp/canada/guide/eql-egl-fra.cfm] (page consultée le 8 décembre 2009).

POINT DE BASCULE. Homophobie chez les immigrants: Québec investira $150000 \$$ [http://www.pointdebasculecanada.ca/breve/2066-quebec-homophobie-chez-lesimmigrants-quebec-investira-150-000.php] (page consultée le 8 décembre 2009).

GOUVERNEMENT DU QUÉBEC (2005). Règlement sur la sélection des ressortissants étrangers, L.R.Q., c. I-0.2, r.5, Québec, Éditeur officiel du Québec.

Tremblay, Pierre J. The Additional Problems of Gay, Lesbian, and Bisexual Youth of Colour. [http://www.sws.soton.ac.uk/gay-youth-suicide/04-gay-youth-of-colour.htm] (page consultée le 8 décembre 2009).

Wong, Alan, et al. (2007). Identités invisibles - Diversité sexuelle des minorités visibles, des communautés culturelles et des personnes bi-spirituelles au Québec, Mémoire présenté à la Commission de consultation sur les pratiques d'accommodement reliées aux différences culturelles, Montréal, octobre. 